\title{
Partially integrable nonlinear equations with one higher symmetry
}

\author{
Alexander V. Mikhailov ${ }^{+*}$ Vladimir S. Novikov\&* and Jing Ping Wang\& \\ + Applied Mathematics Department, University of Leeds, UK \\ \& Institute of Mathematics and Statistics, University of Kent, UK
}

February 8, 2008

\begin{abstract}
In this paper we present a family of second order in time nonlinear partial differential equations, which have only one higher symmetry. These equations are not integrable, but have a solution depending on one arbitrary function.
\end{abstract}

\section{Introduction}

For a long time there has been a general belief that "if a partial differential equation or a system of differential equations has one nontrivial symmetry, then it has infinitely many" [1. Indeed, this statement is true in the case of evolutionary equations, which right hand side is a homogeneous differential polynomial [2]. However, in 1991 Bakirov proposed an example putting this conjecture in doubt [3]. He found that the system

$$
\left\{\begin{array}{l}
u_{t}=u_{x x x x}+v^{2} \\
v_{t}=\frac{1}{5} v_{x x x x}
\end{array}\right.
$$

has a symmetry of order 6 and he also verified using a computer algebra software that it does not have other symmetries up to the order 53. Recently, using $p$-adic analysis, it has been rigorously proven that system (1) has only one higher symmetry and therefore the above conjecture is not valid [4. Furthermore, there exist infinitely many systems of the same type with only one high symmetry. Even a modified conjecture, stating that existence of $n$ higher symmetries for $n$-component system of evolutionary equations implies infinitely many [5] is not valid either - there exists a system, similar to (10) of order 7, which has only two higher symmetries [6].

The Cauchy problem for systems of the form (1) is always solvable no matter how many symmetries the system has. One can solve the second equation and substitute the solution into the first one. The equation obtained becomes a linear inhomogeneous equation, which can be solved using standard techniques.

In this letter we present the following family of partial differential equations $n \geq 2$

$$
u_{t t}=u_{n, t}-\frac{u_{t}}{u}\left(u_{n}-u_{t}\right)+\frac{\left(u_{n}-u_{t}\right)^{2}}{u}-u \partial_{x}^{n}\left(\frac{u_{n}-u_{t}}{u}\right) .
$$

Here and further below we adopt the notation $u_{k} \equiv \partial_{x}^{k}(u)$. The result of our study can be formulated as the following

Theorem 1. For any $n \geq 2$ equation (2) possess a local higher symmetry

$$
u_{\tau}=\frac{1}{u}\left(u_{n}-u_{t}\right)
$$

This symmetry is the only local higher symmetry of equation (2).

Equation (2) is not integrable by any known methods. Nevertheless there is a family of exact solution depending on one arbitrary function.

* On leave, Landau Institute for Theoretical Physics, Moscow, Russia 


\section{Proof of the Theorem and discussion}

There are many equivalent definitions of infinitesimal local higher symmetries for partial differential equations (see for instance [7]). In this paper we adopt the following definition. A partial differential equation $u_{\tau}=G\left(u_{m}, u_{m-1}, \ldots, u, u_{s, t}, u_{s-1, t}, \ldots, u_{t}\right)$ generates a local symmetry of equation (2) if it is compatible with equation (2) [8]. A symmetry is called a higher symmetry of equation (2) if $m>1$ or $s>1$.

Surprisingly enough equation (2) and its symmetry (3) can be rewritten in a very compact form

$$
\begin{gathered}
z_{t}=z_{n}-z^{2}, \\
u_{\tau}=z,
\end{gathered}
$$

where

$$
z=\frac{1}{u}\left(u_{n}-u_{t}\right)
$$

For any $n \geq 2$ equation (44) does not posses higher symmetries and is not integrable by the inverse scattering method or solvable by any other methods. Its trivial solution $z=0$ corresponds to a stationary point of symmetry (5) and provides a nontrivial family of solutions to equation (2). Indeed, $z=0$ implies

$$
u_{t}=u_{n}
$$

The general solution of this linear equation depends on one arbitrary function and it can be found by standard methods.

\section{Proof of the Theorem}

Let us rewrite equation (2) as a system of two evolutionary equations on variables $u$ and $v=u_{t}$ as follows:

$$
\begin{aligned}
& u_{t}=v, \\
& v_{t}=v_{n}-\frac{v}{u}\left(u_{n}-v\right)+\frac{\left(u_{n}-v\right)^{2}}{u}-u \partial_{x}^{n}\left(\frac{u_{n}-v}{u}\right) \equiv H[u, v] .
\end{aligned}
$$

Equation (31) is a symmetry of (2) if and only if system (7) is compatible with

$$
\begin{aligned}
& u_{\tau}=\frac{1}{u}\left(u_{n}-v\right), \\
& v_{\tau}=D_{t}\left(\frac{1}{u}\left(u_{n}-v\right)\right)=\partial_{x}^{n}\left(\frac{u_{n}-v}{u}\right)-\frac{\left(u_{n}-v\right)^{2}}{u^{2}},
\end{aligned}
$$

where $D_{t}$ stands for the operator of total differentiation with respect to $t$ according to (7). Calculating the cross derivatives and expressing the results in terms of variable $z$ (6) we obtain:

$$
\begin{aligned}
& u_{t \tau}=v_{\tau}=z_{n}-z^{2}, \\
& u_{\tau t}=z_{t}=z_{n}-z^{2}
\end{aligned}
$$

and hence $u_{t \tau}=u_{\tau t}$. Analogously

$$
\begin{aligned}
& v_{t \tau}=\partial_{x}^{n}\left(z_{n}-z^{2}\right)-2 z\left(z_{n}-z^{2}\right)-u \partial_{\tau}\left(z_{n}-z^{2}\right)-v z_{\tau}, \\
& v_{\tau t}=\partial_{x}^{n}\left(z_{n}-z^{2}\right)-2 z\left(z_{n}-z^{2}\right) .
\end{aligned}
$$

It is easy to verify that $z_{\tau}=0$ and therefore $v_{t \tau}=v_{\tau t}$ and hence (3) is the symmetry of equation (2).

Let us prove that symmetry (3) is the only local higher symmetry of equation (2). In our proof we shall use elements of the Perturbative Symmetry Approach in the symbolic representation [2, 9 .

Function $H[u, v]$ in the right hand side of the system (7) can be rewritten in the form

$$
H[u, v]=-u_{2 n}+2 u_{n, t}+\sum_{i=1}^{n} f_{i}(u) P_{i}[u, v],
$$

where $f_{i}(u)=\frac{1}{u^{i}}$ and $P_{i}[u, v]$ are polynomials in $v$ and derivatives of $u$ and $v$ with respect to $x$ with constant coefficients, such that

$$
P_{i}[\lambda u, \lambda v]=\lambda^{i+1} P_{i}[u, v], \lambda \in C .
$$


Equation (2) is a homogeneous equation, therefore polynomials $P_{i}$ are homogeneous polynomials of the weight $2 n$, i.e. $W\left(P_{i}\right)=2 n P_{i}$, where

$$
W=\sum_{k=1}^{\infty} k u_{k} \frac{\partial}{\partial u_{k}}+\sum_{k=0}^{\infty}(k+n) v_{k} \frac{\partial}{\partial v_{k}} .
$$

Function $H[u, v]$ can be treated as a differential polynomial in $v$ and derivatives of $u, v$ with coefficients being functions of $u$ only. The symbolic representation of such polynomials have been defined and studied in [10]

To prove the second statement of the theorem we will need only a few first terms of the function $H[u, v](9)$ :

$$
\begin{aligned}
& H[u, v]=-u_{2 n}+2 v_{n}+ \\
& +f_{1}(u)\left(\partial_{x}^{n}\left(u u_{n}-u v\right)-u u_{2 n}+u_{n}^{2}+u v_{n}-3 u_{n} v+2 v^{2}\right)+R[u, v],
\end{aligned}
$$

where $R[u, v]=\sum_{i \geq 2} f_{i}(u) P_{i}[u, v]$. Then in the symbolic representation system (7) can be rewritten as

$$
\begin{aligned}
& u_{t}=\hat{v} \\
& \left.v_{t}=-\hat{u} k_{1}^{2 n}+2 \hat{v} q_{1}^{n}+f_{1}(u) \circ\left[\hat{u}^{2} a_{1}\left(k_{1}, k_{2}\right)+\hat{u} \hat{v} a_{2}\left(k_{1}, q_{1}\right)+2 \hat{v}^{2}\right)\right]+\hat{R}[u, v],
\end{aligned}
$$

where

$$
\begin{aligned}
& a_{1}\left(k_{1}, k_{2}\right)=\frac{1}{2}\left[\left(k_{1}+k_{2}\right)^{n}\left(k_{1}^{n}+k_{2}^{n}\right)-\left(k_{1}^{n}-k_{2}^{n}\right)^{2}\right] \\
& a_{2}\left(k_{1}, q_{1}\right)=q_{1}^{n}-\left(k_{1}+q_{1}\right)^{n}-3 k_{1}^{n}
\end{aligned}
$$

and $\hat{R}[u, v]$ stands for the symbolic representation of $R[u, v]$.

It is easy to verify that the most general form of a higher symmetry $(m \geq 2)$ of system (7) in the symbolic representation is:

$$
\begin{aligned}
u_{\tau}= & g_{1}(u) \circ \hat{u} k_{1}^{m}+g_{2}(u) \circ \hat{v} q_{1}^{m-n}+\hat{u}^{2} A_{1}\left(k_{1}, k_{2} ; u\right)+ \\
& +\hat{u} \hat{v} A_{2}\left(k_{1}, q_{1} ; u\right)+\hat{v}^{2} A_{3}\left(q_{1}, q_{2} ; u\right)+\hat{S}[u, v] \equiv G, \\
v_{\tau}= & D_{t}(G)
\end{aligned}
$$

Here $A_{i}(x, y ; u)$ are polynomials in $x, y$ with coefficients, depending on $u$. Polynomials $A_{i}(x, y ; u)$ satisfy the condition $A_{i}(0, y)=0, i=1,2$. The remainder $\hat{S}[u, v]$ stands for the terms of higher nonlinearity in $v$ and $x$-derivatives of $u, v$.

Lemma 1. If (12) is a symmetry of equation (11) then $g_{1}(u), g_{2}(u)$ are not equal to zero simultaneously.

Sketch of the proof: Let us assume that symmetry (12) does not have linear terms (in $\hat{u}, \hat{v})$ and starts with the terms of order $s>1$ :

$$
u_{\tau}=\sum_{p=0}^{s} A_{p}\left(k_{1}, k_{2}, \ldots, k_{p}, q_{1}, q_{2}, \ldots, q_{s-p} ; u\right) \hat{u}^{p} \hat{v}^{s-p}+\text { higher order terms }
$$

Then it follows from the compatibility conditions of (11) and (12) that the coefficients $A_{p}\left(k_{1}, k_{2}, \ldots, k_{p}, q_{1}, q_{2}, \ldots, q_{s-p} ; u\right)$ satisfy a linear system of homogeneous algebraic equations. This system has only a trivial solution $A_{p}=0, p=0, \ldots, s$ since the determinant of the corresponding matrix is not identically vanishing. Lemma is proved $\mathbf{\square}$

The case $m=n$ corresponds to symmetry (3). In this case $A_{i}(x, y ; u)=0$ and $g_{1}(u)=-g_{2}(u)=\frac{1}{u}$.

Let $m \neq n$. From the compatibility conditions of (11) and (12) it follows that

$$
A_{3}\left(q_{1}, q_{2} ; u\right)=\frac{1}{2} f_{1}(u) g_{2}(u) \frac{q_{1}^{m-n}+q_{2}^{m-n}-2\left(q_{1}+q_{2}\right)^{m-n}}{q_{1}^{n}+q_{2}^{n}-\left(q_{1}+q_{2}\right)^{n}} .
$$

Polynomial $q_{1}^{n}+q_{2}^{n}-\left(q_{1}+q_{2}\right)^{n}$ does not divide $q_{1}^{m-n}+q_{2}^{m-n}-2\left(q_{1}+q_{2}\right)^{m-n}$ if $m \neq n$ and therefore $A_{3}\left(q_{1}, q_{2} ; u\right)$ does not represent a symbol of a differential polynomial with $u$-depended coefficients. It implies that $g_{2}(u)=0$.

Having $g_{2}(u)=0$ we find (from the compatibility conditions) that

$$
A_{2}\left(k_{1}, q_{1} ; u\right)=f_{1}(u) g_{1}(u) \frac{k_{1}^{m}+q_{1}^{m}-\left(k_{1}+q_{1}\right)^{m}}{k_{1}^{n}+q_{1}^{n}-\left(k_{1}+q_{1}\right)^{n}} .
$$


For $s>1$ the polynomial $K_{s}(x, y)=x^{s}+y^{s}-(x+y)^{s}$ can be factorized as

$$
K_{s}(x, y)=x y T_{s}(x, y), \quad T_{s}(0, y) \neq 0
$$

Hence

$$
A_{2}\left(k_{1}, q_{1} ; u\right)=f_{1}(u) g_{1}(u) \frac{T_{m}\left(k_{1}, q_{1}\right)}{T_{n}\left(k_{1}, q_{1}\right)} .
$$

It follows from (13) $A_{2}\left(0, q_{1} ; u\right)=0$ only if $g_{1}(u)=0$. It follows from the Lemma, that a symmetry without a linear part does not exist. The theorem is proved

Acknowledgements. VSN is funded by a Royal Society NATO/Chevening fellowship on the project Symmetries and coherent structures in non-evolutionary partial differential equations. AVM and VSN thank the RFBR Grant No. 02-01-00431 for partial support.

\section{References}

[1] A. S. Fokas. A symmetry approach to exactly solvable evolution equations. J. Math. Phys., 21(6):1318-1325, 1980 .

[2] Jan A. Sanders and Jing Ping Wang. On the integrability of homogeneous scalar evolution equations. $J$. Differential Equations, 147(2):410-434, 1998.

[3] I.M. Bakirov. On the symmetries of some system of evolution equations. Technical report, Akad. Nauk SSSR Ural. Otdel. Bashkir. Nauchn. Tsentr, Ufa, 1991.

[4] Frits Beukers, Jan A. Sanders, and Jing Ping Wang. One symmetry does not imply integrability. J. Differential Equations, 146(1):251-260, 1998.

[5] A.S. Fokas. Symmetries and integrability. Studies in Applied Mathematics, 77:253-299, 1987.

[6] Peter H. van der Kamp and Jan A. Sanders. Almost integrable evolution equations. Selecta Math. (N.S.), $8(4): 705-719,2002$.

[7] A. V. Mikha1̆lov, A. B. Shabat, and V. V. Sokolov. The symmetry approach to classification of integrable equations. In What is integrability?, Springer Ser. Nonlinear Dynamics, pages 115-184. Springer, Berlin, 1991.

[8] R. Hernández Heredero, A.B. Shabat and V.V. Sokolov, "A new class of linearizable equations", submitted Physics Lett A, published in arXiv:nlin.SI/0301001 v1, 3 Jan 2003.

[9] A.V. Mikhailov, V.S. Novikov, "Perturbative Symmetry Approach", Journal of Physics A, Vol 35, pp. 4775-4790, 2002.

[10] Jan A. Sanders, Jing Ping Wang. On the integrability of non-polynomial scalar evolution equations. J. Differential Equations, 166 (2000), 1, 132-150 